\title{
TechDoc - System for Queue Management of Pharmacies and Online Consultation of Doctors
}

Avali Banerjee| Deepak Pandey| Mamata Singh| Atrayee Gayen|Kashish Rani|Prafulla Kumar Sahani| Ayushi Jaiswal

Department of ECE, Guru Nanak Institute of Technology,Kolkata, West Bengal, India

To Cite this Article

Avali Banerjee,; Deepak Pandey,; Mamata Singh,; Atrayee Gayen,; Kashish Rani,; Prafulla Kumar Sahani \& Ayushi Jaiswal. TechDoc - System for Queue Management of Pharmacies and Online Consultation of Doctors. International Journal for Modern Trends in Science and Technology 7, 21-25 (2021).

\section{Article Info}

Received on 28-April-2021, Revised on 22-May-2021, Accepted on 28-May-2021, Published on 04-June-2021.

\section{ABSTRACT}

A TechDoc system has been developed for online management of pharmacies and consultation of doctors and presented in this paper. TechDoc serves the purpose of queue management outside pharmacies, by providing a delivery system of online ordered medicines from registered pharmacies nearby to customers' residence; checking of symptoms using the free symptom checker that can diagnose a large number of diseases and gives relevant details on remedies for the diagnosed disease along with a video for home remedies of the particular disease; booking an appointment with a physical practitioner through the doctor's consultation section in case the user is unsatisfied with the home remedies generated by the system. All these facilities are implemented into a single platform - a website accessible by any device, an android application especially for android phones and an IVR system to be accessed by the users who don't have access to high end devices or internet connection. This innovative implementation helps in easy availability of essential services like, ordering of medicines, provisional diagnosis of curable disease and consultation of doctors, online and promoting social distancing.

KEYWORDS:Queue management, pharmacies, online consultation, medicines, post-pandemic world, IVR

\section{INTRODUCTION}

The outbreak of the SARs Covid-19 has led to a global pandemic situation. Implementation of innovative ideas with the help of technologies involving website development, application development and IVR development can make sustainability of common people in the new normal world easier.

In the present scenario, social distancing is an important practice and TechDoc website and android application promotes social distancing by the queue management system developed for the pharmacies. A new user needs to register either through website or application or IVR. The specially designed feature for ordering from pharmacies allows the users to purchase medicines from their nearby registered pharmacies. On receiving the orders from the users end the pharmacies will allot a specific time for preparing the orders. Once the orders are prepared the users get notification to pick up their orders. Following this procedure, TechDoc aims at managing the unnecessary crowd at pharmacies.

The users need not physically visit physicians They can initially have a diagnosis through the symptom checker and if they are not satisfied, they can book an appointment to consult any physician. This facility not only reduces unnecessary gatherings but also aims at improving online healthcare facilities. 


\section{METHODOLOGY}

\section{SYMPTOM CHECKER FOR DISEASES}

This work contains a symptom checker which checks symptoms entered by the user and gives relevant diagnosis and also provides video recommendation of home remedies for the diagnosed diseases (curable through home remedies). If the disease is serious or incurable, then it provides a relevant helpline number of hospitals.

\section{QUEUE MANAGEMENT SYSTEM FOR PHARMACIES}

The 2nd aspect of this work is the queue management system. The user must register as a customer by entering their name, address, and mobile number. The registered customers can check the availability of their needed medicines in the nearby registered pharmacies and place their orders.

The application calculates the assumed time after which the customer's order is ready according to the number of accumulated orders and gives the customer a time slot and receives a notification when the order is ready to be picked up.

\section{CONSULTATION OF DOCTORS}

In doctors' consultation section, different doctors can register themselves by providing required details. The registered users can easily book an in-person appointment or consult them via online mode according to their necessity.

\section{UPDATING AND MANAGEMENT OF DATA BY CUSTOMERS, PHARMACISTS, AND DOCTORS}

This application will provide a friendly environment for the users, pharmacists, and doctors to manage their profiles and update any required information.

The customers can easily check the availability of their needed items and pharmacists can easily update the status of any item. It will be a low-cost implementation, created solely to help the cause of this pandemic.

We will provide details of the doctor and the customer must book the appointment using the application.

There will be an IVR (Interactive Voice Response) system so that people who don't have access to the internet or smartphones can use the services.

Fig. 1 shows the flowchart. As an initial step the user can select from any of the three interface website, android application or IVR as shown in Fig. 1 (a) and (b).
Using the selected interface, user must check if he/she is registered to the portal or not. Already registered users can simply login with their login credentials to the website or android application or start using the services of IVR.

While using the IVR, users must select language in which they want to hear the instructions.

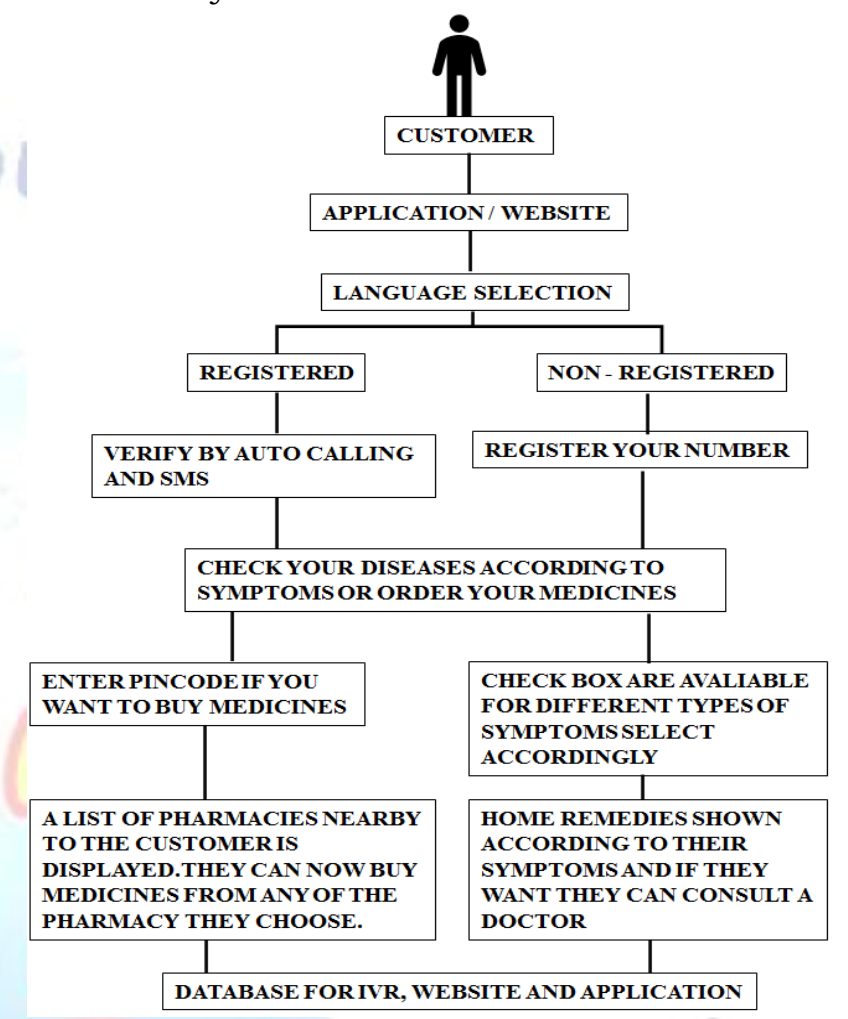

(a)

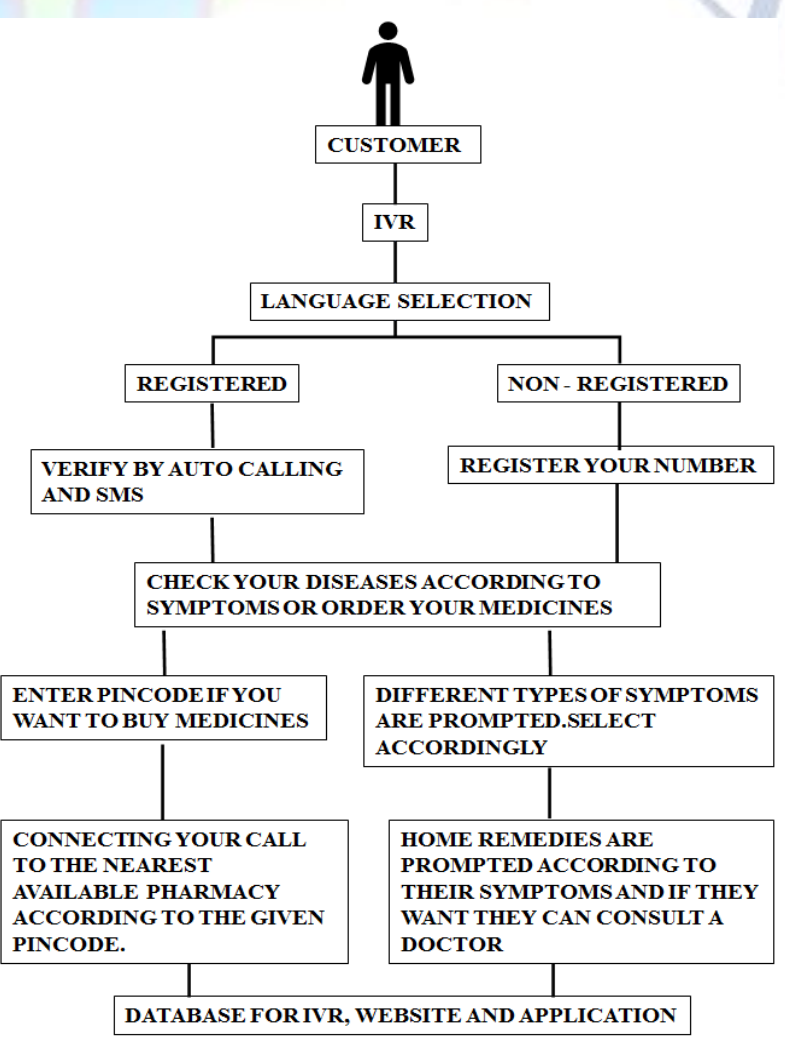

(b) 


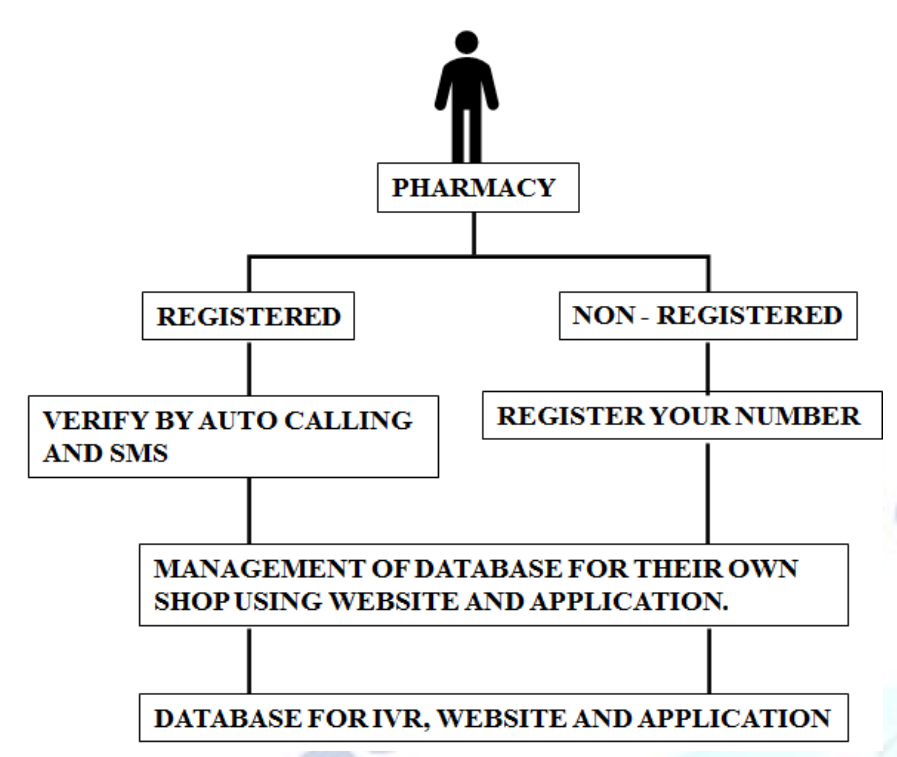

(c)

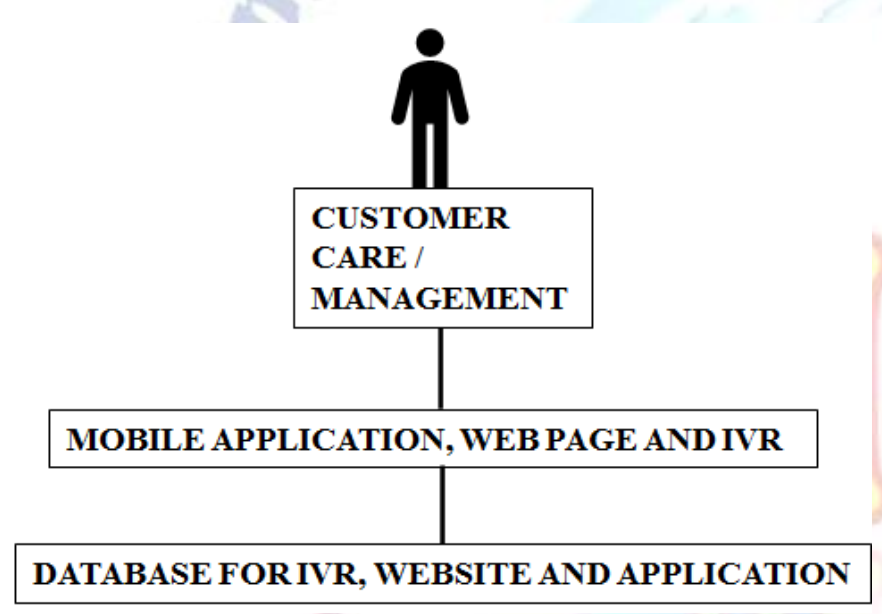

(d)

Fig. 1. Flowchart (a) Customer using android application orwebsite (b) Customer using IVR (c)

Pharmacy (d) Customer care / Management

After successful registration of non-registered users and after language selection in IVR, verification is done by auto calling or SMS.A number of features are provided by the system and they are

\section{Symptom Checking}

Using the website or the application the user gets to enter his/her symptoms through symptom checker check boxes. The symptom checker diagnoses the most probable disease and displays the home remedies to cure the disease along with a video for the home remedy. Using the IVR the user must select the symptoms by pressing keys and the relevant home remedy will be prompted.

\section{Consultation}

If the user is not satisfied with the symptom checker the can opt for online consultation of doctors.

\section{Ordering from pharmacies}

Initially users need to enter their area pincode. They can then order medicines from nearby registered pharmacies by selecting the relevant option in IVR and the call will be auto connected to the pharmacies. In the website and application, they can directly choose the items they want to order and if they are available in the user selected pharmacy the order will be placed. After the order is prepared the user gets a notification to pick up the order from the pharmacy.

The registered pharmacies get to use a different website and application for managing and updating their products on the main portal. This also helps them to receive orders and allot time for preparing the orders.

All the database from the application, website and IVR are fetched, managed, and maintained by the backend management team.

\section{RESULT}

This work can be helpful in improving or developing the upcoming diagnosis system of our country. Due to the pandemic many people are taking measures and are making themselves go out of their houses what the minimum reasons to setup out of their houses. This work can provide them opportunities to consult with your doctor or by any medicines by the ease of being at home. Due to the IVR system provided in this work, the people who don't have proper internet connection or don't use smartphones can also use our facilities without any issue. Fig. 2 ( $a, b, c, d$, e, and f) shows the screenshots of the website.

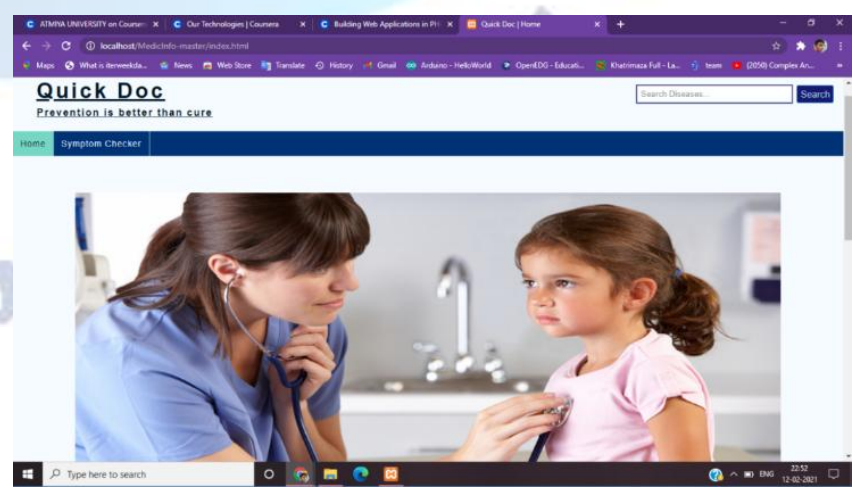

(a) 


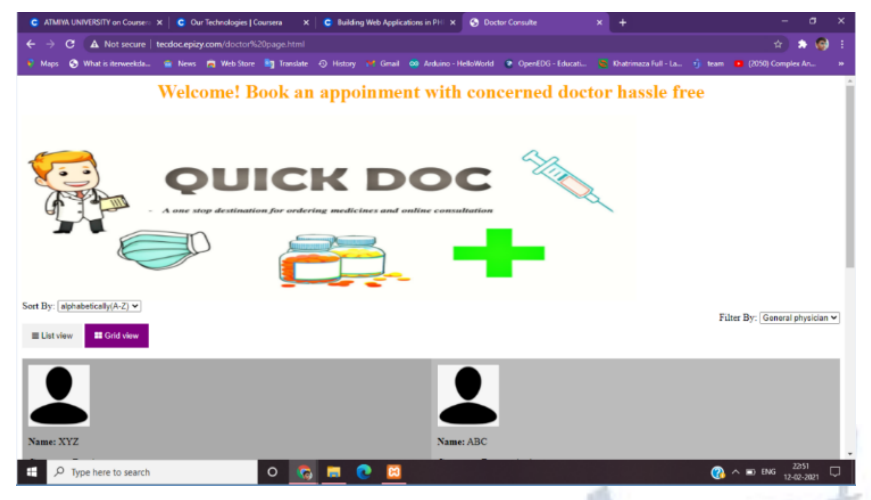

(b)
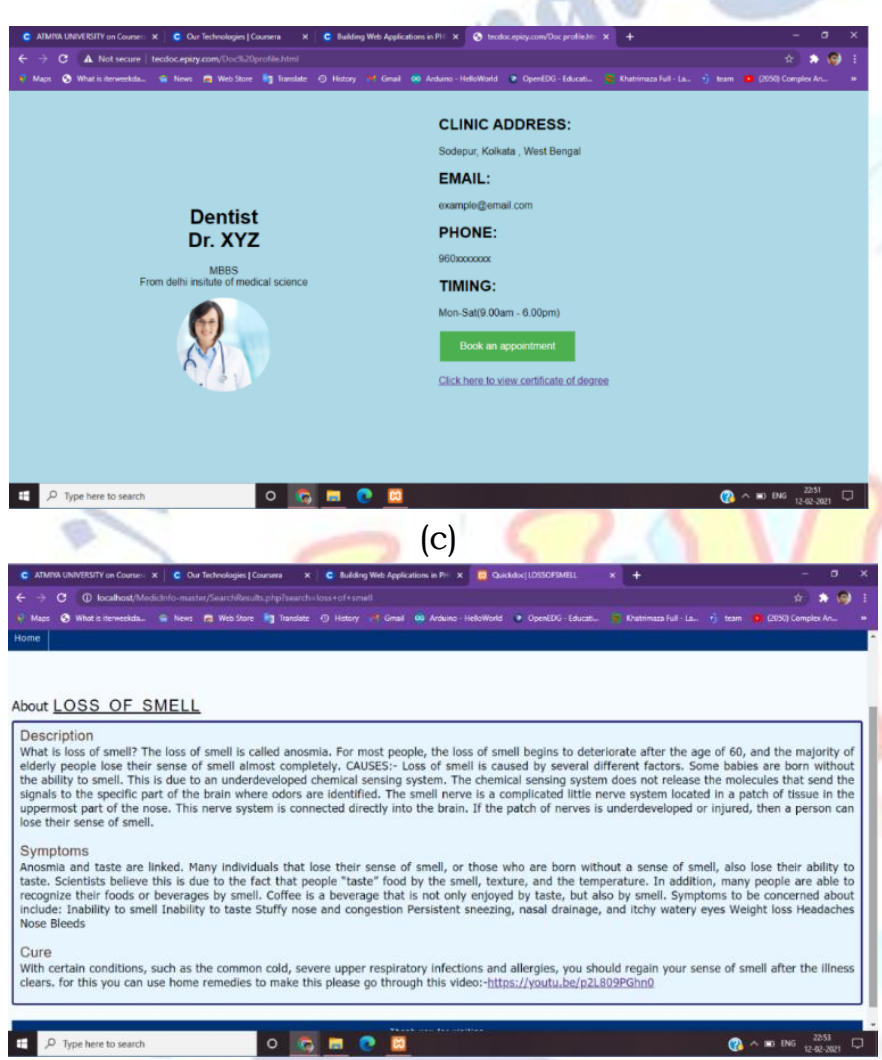

(d)
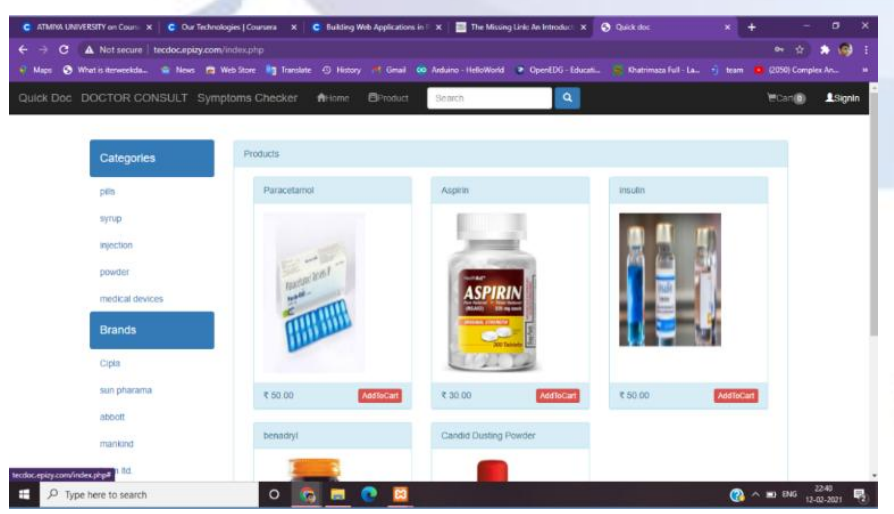

(e)
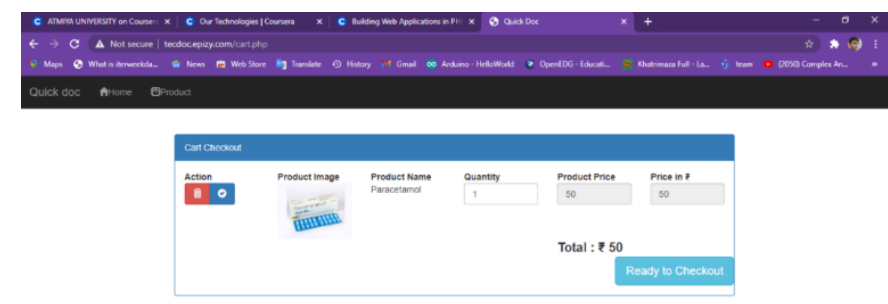

-

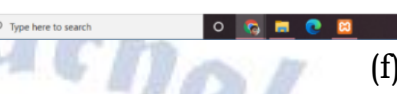

Fig. 2. Screenshots of the website

\section{CONCLUSION}

The overall system has been seen to function efficiently and is supposedly beneficial to users for the current pandemic situation.

The queue management system is essential and efficient for managing crowd in front of pharmacies, as it will allot a specific time for picking up the prepared orders to the consumers.

The symptom checker is a free of cost and user-friendly interface that not only checks the symptoms to find the disease but also generates details on remedies along with a tutorial video for home remedies.

The users, who won't be satisfied with the symptom checker and system generated remedies can opt for online facilities for consulting doctors. The users can select the category of physician they wish to consult and accordingly fill up a form to book an appointment.

An IVR system is created keeping in mind the digital divide environment. This ensures that users without high end devices like computers and smartphones are also able to use all the services easily and comfortably.

The initial registration of the pharmacies according to different localities, to the system is a challenging task. Proper updating of available medicines in pharmacies, in the system is to be done time to time. The remedies provided by the symptom checker or advice given by the doctor may not satisfy every customer. These are few probable disadvantages related to the usability and functionality of the system.

\section{FUTURE SCOPE}

This application will be helpful to sustain life in the new normal world as it will be beneficial to maintain social distancing in places like the hospitals, clinics, and pharmacies. 
A person doesn't have to go across different pharmacies to find a single medicine. One can book an appointment with the desired doctor without visiting the clinic or hospital. People can easily take the advice of doctors from their residence through online mode.

If a person is not sure about the health issues and doesn't want to consult a doctor in hurry, then they can use the symptoms checker to find the possibility of disease.

\section{REFERENCES}

[1] Sarah Aldossary, Turki Alanzi: Patients' opinions about the use of Snapchat in medical consultations in Saudi Arabia: 2020 19th International Symposium on Distributed Computing and Applications for Business Engineering and Science (DCABES), 16-19 Oct. 2020, Xuzhou, China. DOI: $10.1109 /$ DCABES50732.2020.00040

[2] Tan KhianChuan, Nasriah Zakaria, Mohd Azam Osman, User requirements for online consultation in Cancer Patient Portal, 2010 IEEE EMBS Conference on Biomedical Engineering and Sciences (IECBES), 30 Nov.-2 Dec. 2010, Kuala Lumpur, Malaysia, DOI: $10.1109 /$ IECBES.2010.5742268

[3] Csaba Jenei; TamásIvánfai; BálintKőszegi; ZsoltKőszegi, Webbase Online Consulting System for quasi real time consultations of cardiac images, Computing in Cardiology 2013, 22-25 Sept. 2013, Zaragoza, Spain.

[4] WH Yan, "On consulting fees: pay and arrears?", Popular Psychology, vol. 12, pp. 4-6,Dec. 2014.

[5] ChunyuYisheng,Baidubaike,2011,[online]Available:http:// baike.baidu.com

[6] X Cao, Y Liu, Z Zhu, J Hu and X. Chen, "Online selection of a physician by patients: Empirical study from elaboration likelihood perspective [J]", Computers in Human Behavior, vol. 73, pp. 403-412, 2017

[7] $\mathrm{H}$ Wu and N. Lu, "Online Written Consultation Telephone Consultation and Offline Appointment: An Examination of the Channel Effect in Online Health Communities [J]", International Journal of Medical Informatics, vol. 107, pp. 107-119, 2017.

[8] M Tanis, T Hartmann and F T. Poel, "Online health anxiety and consultation satisfaction: A quantitative exploratory study on their relations [J]", Patient Education \& Counseling, vol. 99, no. 7, pp. 1227-1232, 2016.

[9] J Lv and JP Wang, "Doctor's assistant - Mobile Medical App", Medical Journal of Beijing Military Region, vol. 3, pp. F0003, Mar. 2015.

[10] ZR Luo, A Guo and JD Yan, "Influence of Payment Method Evolution on: Diagnosis and Treatment Processing", Chinese Hospital Management, vol. 3, pp. 19-21, Mar. 2015.

[11] Y. Chen, L. Yang, M. Zhang and J. Yang, "Central or peripheral? Cognition elaboration cues' effect on users' continuance intention of mobile health applications in the developing markets", International Jounal Medical Informatic, vol. 116, pp. 33-45, 2018.

[12] J Mueller, C Jay, S Harper, A Davies, J Vega and C. Todd, "Web Use for Symptom Appraisal of Physical Health Conditions: A Systematic Review", J Med Internet Res, vol. 19, no. 6, pp. e202, 2017

[13] Doctor consultant:-https://www.doconline.com/online-doctor-co nsultation

[14] Symptoms cheaker:-https:/ / familydoctor.org/your-health-resources / health-tools/symptom- checker/ 